\title{
The few scales of nuclei and nuclear matter
}

\author{
A. Delfino ${ }^{a}$, T. Frederico ${ }^{\text {b }}$, V. S. Timóteo ${ }^{\text {c }}$, and Lauro Tomio ${ }^{d}$ \\ ${ }^{a}$ Instituto de Física, Universidade Federal Fluminense, 24210-900 Niterói, RJ, \\ Brasil \\ ${ }^{\mathrm{b}}$ Departamento de Física, Instituto Tecnológico de Aeronáutica, CTA, 12228-900, \\ São José dos Campos, Brasil \\ ${ }^{\mathrm{c}}$ Centro Superior de Educação Tecnológica, Universidade Estadual de Campinas, \\ 13484-370, Limeira, SP, Brasil \\ ${ }^{\mathrm{d}}$ Instituto de Física Teórica, Universidade Estadual Paulista, 01405-900, São \\ Paulo, Brasil
}

\begin{abstract}
The well known correlations of low-energy three and four-nucleon observables with a typical three-nucleon scale (e.g. the Tjon line) is extended to light nuclei and nuclear matter. Evidence for the scaling between light nuclei binding energies and the triton one are pointed out. We show that the saturation energy and density of nuclear matter are correlated to the triton binding. From the available systematic nuclear matter calculations, we verify the existence of bands representing these correlations.
\end{abstract}

PACS 21.45.+v, 21.65.+f, 21.30.Fe

Key words: Scaling, nonrelativistic few-body systems, nonrelativistic nuclear matter

Two-nucleon interactions are typically constructed to fit scattering data and deuteron properties. When such interactions are used to calculate three-nucleon observables, the results exhibit some discrepancies [1]. Basically, they are explained as originated from different strengths of the two-nucleon tensor force and short-range repulsions, provided that all realistic two-nucleon interactions have the correct one-pion exchange tail. In four-nucleon bound state $\left({ }^{4} \mathrm{He}\right)$ calculations the discrepancies still remain. But, at least, they are correlated, as seen in the binding energies of ${ }^{4} \mathrm{He}\left(B_{\alpha}\right)$ and triton $\left(B_{t}\right)$, which lie on a very narrow band [2], obtained when the short-range repulsion of the nucleonnucleon interaction is varied while two-nucleon informations (deuteron and scattering) are kept fixed. This correlation is known as Tjon line [2]. $B_{\alpha}$ and $B_{t}$ follows an almost straight line in the range of about $1-2 \mathrm{MeV}$ of variation 
of the triton binding energy around the experimental value. As the long-range two-nucleon scales we have the deuteron binding energy $\left(B_{d}\right)$ and the singlet virtual-state energy $\left(B_{v}\right)$.

Two-body short-ranged interactions, supporting very low two-body binding energy and/or large scattering lengths, when used to calculate three-body systems, approach what we call the universal Thomas-Efimov limit [3]. By trying to find the range $r_{0}$ of the two-nucleon force, Thomas [4] showed that when $r_{0} \rightarrow 0$, while the two-body binding energy $B_{2}$ is kept fixed, the three-body binding energy goes to infinity (Thomas collapse). Much latter, Efimov [5] showed that, in the limit $B_{2}=0\left(r_{0} \neq 0\right)$ the number of three-body bound states is infinite with an accumulation point at the common two- and threebody threshold. Note that both the Thomas and Efimov effects are claimed to be model independent, since they are due to a dynamically generated effective three-body potential acting at distances outside the range of the two-body potential. These apparently different effects are related to the same scaling mechanism, as shown in Ref. [3]. In other words, the Thomas effect appears when $r_{0}$ is much smaller than the size of the two-body system (which is of the order of the scattering length $|a|$ ), while the Efimov effect arises for $|a|>>r_{0}$. Therefore, what matters for both effects is the same condition: $|a|>>r_{0}$ or the ratio $|a| / r_{0}>>1$. In terms of the two-body energies this is translated to $\sqrt{\left(m\left|B_{2}\right| / \hbar^{2}\right)} r_{0}<<1$ ( $m$ the boson mass). One would expect that the Thomas-Efimov effect is manifested in weakly-bound quantum few-body systems which are much larger in size than the corresponding two-body effective range. Notice that a zero binding energy for a free two-body system is not known in nature. But, nowadays it was shown that, for trapped ultracold gases of certain atomic species, it is possible to adjust the two-body scattering length at very large values, using Feshbach resonance techniques, by tuning the external magnetic field [6]. In this case, it is expected that the Thomas-Efimov effect can be manifested [7].

The deuteron and triton may be viewed as low energy systems with large size scales in which the range of the potential is smaller than the corresponding healing distances of the wave functions, leading the nucleons to have a high probability to be outside of the interaction range. Then, the low-energy properties of these systems can be studied with models that minimally includes the physics of the Thomas-Efimov effect, as in the case of a few-body model with renormalized pairwise s-wave zero-range force [8]. This approach shows that all the low-energy properties of the three-body system are well defined in the model, once one three-body scale and the two-body low-energy observables are given. As a consequence, correlations between two three-body $s$-wave observables are expected to appear in model calculations with short-ranged interactions. Along this line, some previous works (see 9] and references therein) have studied weakly-bound halo states in exotic nuclei as well as possible Efimov 
states for He-He-Alkali molecules.

The scaling of three-nucleon observables with the triton binding energy corresponds to universal behaviors found when a three-body scale is varied. For example, the Phillips plot [10] of the neutron-deuteron doublet scattering length, as a function of the triton energy is nowadays one of the universal scalings found in the three-nucleon system [11,12. In general, it is observed for nuclear and molecular weakly bound three-body systems [13,14,15] the scaling of observables with the three-body binding energy.

The Thomas-collapse of the three-body energy in systems of maximum wavefunction symmetry implies the existence of a three nucleon scale (identified with the triton binding energy) governing the short-range behavior of the wave-function. Four nucleons can also form a state of maximum symmetry, allowing in principle the collapse of such configuration, independently of the three-nucleon collapse [8]. This is under discussion and it is suggested in [16] that the four-body scale is not independent of the three-body one. However, in their work it was introduced a three-body force to stabilize the shallowest three-body state, against the variation of the cut-off. The three-body interaction can be attractive or repulsive and their conclusion lies on the repulsive sector. We note that the attractive part indicates a possible independent behavior of the four body ground-state energy from the three-body one. Certainly this point merits further discussions and so far, we think, it is still open the possibility of a four-body scale. Anyway, as the nucleon-nucleon interaction is strongly repulsive at short range and therefore the probability of four nucleons to be simultaneously in a volume $\sim r_{0}^{3}$ is quite small, presumably the four-nucleon scale itself has much less opportunity to be evidenced in realistic nuclear models. Indeed, this is indicated by the existence of the Tjon line. Due to that, as we will see later, the four-nucleon binding energy is eliminated in favor of the triton binding energy. In this respect it is worthwhile to note that Platter et al. extended the effective field theory framework applied to fourbosons [16] to calculate the ${ }^{4} \mathrm{He}$ binding energy by controlling the triton energy through a repulsive effective three-nucleon force. Within their approach [17] the Tjon line is reproduced.

In a nuclear scenario dominated by an interaction with a range smaller than the nucleon-nucleon scattering lengths, and considering the triton and ${ }^{4} \mathrm{He}$ nuclear sizes yet larger than the force range, the picture of nuclei would be of a many-body system with the wave-function being an eigenfunction of the free Hamiltonian almost everywhere. The Pauli principle allows only up to four nucleons at the same position, forbidding certain particular configurations with overlap of more particles. If more than four particles are allowed to overlap, it would imply that the asymptotic information from the interaction of the cluster would go beyond of those already fixed by the low-energy observables of two, three and four nucleons. By some unknown reason the parameters 
of Quantum Chromodynamics are close to this limit. It was conjectured in Ref. [18] that a small change in the light quark masses away from their physical values could put the deuteron and the singlet virtual state at zero binding energy, and therefore the above idealized picture of the nuclear systems could not be far from reality. It is quite amazing thinking that nuclear wave functions could heal much beyond the interaction range. Therefore, the details of the long wavelength structure of nuclei are given by the free Hamiltonian and by few-nucleon scales, which determine the wave function at short distances. The universal behavior of the scaling functions are due to that.

If one wonders about the neutron matter within a non-relativistic quantum framework, in the limit of a zero-range force, we could say that the only scale in this case is the neutron-neutron scattering length. Therefore, the binding energy of neutron droplets will be strongly correlated to that quantity, which is the only physical scale in this situation allowed by the Pauli principle. This discussion has been performed in the context of three neutron systems [19]. Moreover, it was concluded in Ref. [20] that stable tetra-neutron droplets would imply a major change in the neutron-neutron scattering length.

Another example of the dominance of only two-body scale appears in threeboson systems in two dimensions, where the Thomas-Efimov effect is absent [3]. In this case, only two-body low energy scales are enough to define the many-body properties in the limit of a zero-range interaction. The low-energy properties of a many-body system of spin-zero particles in two dimensions will be sensitive only to the two-boson binding energy. Even in the case where bosons are trapped, since the essential singularity of the point-like configuration is not affected by the confining force as the harmonic one.

For the sake of generality, we start with the observables $B_{d}, B_{v}, B_{t}$ and $B_{\alpha}$ as the scales determining the asymptotic properties of nuclei [9]. Then, in the limit of a zero-range interaction, we write the binding energy of a nucleus with mass number $A$ and isospin projection $I_{z}$, considering isospin breaking effects, as

$$
B_{\left(A, I_{z}\right)}=A B_{t} \mathcal{B}\left(\beta_{v}, \beta_{d}, \beta_{\alpha}, A, I_{z}\right)
$$

where $\beta_{a}=B_{a} / B_{t}$ with $a=v, d$ and $\alpha$.

According to the Tjon line, $\beta_{\alpha}$ remains approximately constant for a variety of two-nucleon potentials and the parametrization of the numerical results, given in $\mathrm{MeV}$, for several two-nucleon potentials is

$$
B_{\alpha}=4.72\left(B_{t}-2.48\right),
$$

which for $B_{t}^{e x p}=8.48 \mathrm{MeV}$ gives $B_{\alpha}^{e x p}=28.32 \mathrm{MeV}$. Using (2) in (1), 


$$
R_{\left(A, I_{z}\right)}=B_{\left(A, I_{z}\right)} / A=B_{t} \mathcal{R}\left(B_{t}, A, I_{z}\right),
$$

where in the scaling function $\mathcal{R}_{\left(A, I_{z}\right)}$ the values of $B_{d}$ and $B_{v}$ are fixed to the experimental values. The dependence of $B_{\alpha}$ with $B_{t}$ for realistic nucleonnucleon potentials is given by Eq. (2).

Equation (3) generalizes the concept of the Tjon line to nuclei. Recent calculations using the AV18 nucleon-nucleon potential plus three-body forces 21] show that there is a systematic improvement of the binding energy results for $\mathrm{He}, \mathrm{Li}, \mathrm{Be}$ and $\mathrm{B}$ isotopes simultaneously with the triton binding energy, when models are tuned to fit $B_{t}$. It is important to note that these AV18 calculations have at least two three-body parameters that are fitted to $B_{t}$ and nuclear matter saturation properties. Consequently, one could argue that such calculations cannot provide evidence for one-parameter correlation. The fitting to nuclear matter calculation presumably is not that important for light nuclei in view of the dominance of the triton binding (or three-body correlations) in the four-nucleon bound state as given by the Tjon line. Therefore, it is reasonable to think that three-body correlations are quite important for light-nuclei, since that even for the alpha particle where the nucleons are in a very compact configuration this occurs. In our opinion, the fitting of nuclear matter saturation properties has more to do with the approximations done in nuclear matter calculations. The three-body potential should be somewhat tuned, which is, probably, not so important for light nuclei once the triton binding attains its physical value.

For nuclear matter properties calculation using a variety of two-nucleon potentials, in which the tensor strength was varied but the deuteron binding energy was kept fixed, it was shown that these interactions cannot quantitatively account for nuclear saturation [22]23]. Coester et al. 22] observed that, in an energy versus density plot, the saturation points of nuclear matter obtained by employing different realistic potentials are located along a band (Coester band). Also, in a relativistic framework it was observed such strong correlation [24]. The displayed nuclear matter binding energy $\left(B_{A} / A \equiv B_{(A, 0)} / A\right)$ versus saturation density $\left[\rho_{o}=(2 / 3) k_{F}^{3} / \pi^{2}\right.$, with $k_{F}$ the Fermi momentum] results are within a narrow band [22]. The observation given in Ref. [22] have been studied by many other authors that have used nuclear matter binding energies and saturation densities from different two-nucleon interactions. The main argumentation, as also in the case of three-nucleon calculations, is that this effect comes from different strengths of the two-nucleon tensor force and short range repulsion, which changes the triton binding energy, while keeping fixed the low-energy two-body scales.

Basically, nuclear matter saturates due to the composed repulsive and attractive short-range two-nucleon potential. Since, it may also be seen as a typical low-energy problem, it is natural to question whether any connection exists 


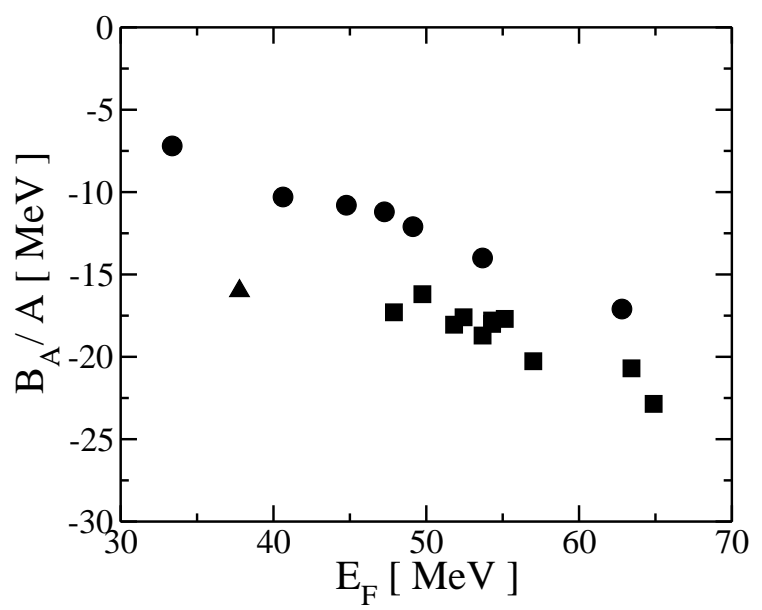

Fig. 1. Infinite nuclear matter binding energy as a function of $E_{F}$ extracted from Ref. [25] (solid circles and squares). The squares includes the single particle contribution in the continuum. The full triangle is given by the empirical values.

between the proper few-body scales, $B_{d}, B_{v}$ and $B_{t}$ with those of the manybody problem, like the $B_{A} / A$ and the Fermi energy $E_{F}=\hbar^{2} k_{F}^{2} /\left(2 m_{N}\right)$. For light nuclei there is strong evidences of scaling between $B_{d}, B_{v}$ and $B_{t}$ as expressed by Eq. (3) .

Here we are arguing that the scales of nuclear matter, $B_{A} / A$ and $E_{F}$, are determined by $B_{d}, B_{v}$ and $B_{t}$. Therefore, we suppose that going to the infinite isospin symmetrical nuclear matter, $A \rightarrow \infty$ and $I_{z}=0$, the limit

$$
\begin{aligned}
\frac{B_{A}}{A} & \doteq \frac{B_{t}}{A} \lim _{A \rightarrow \infty} \mathcal{B}\left(\beta_{v}, \beta_{d}, \beta_{\alpha}, A, I_{z}=0\right) \\
& =B_{t} \mathcal{G}\left(\beta_{v}, \beta_{d}, \beta_{\alpha}\right)
\end{aligned}
$$

is well defined and expresses the correlation between the binding energy of the nucleon in nuclear matter with the few-nucleon scales. The Fermi energy

$$
E_{F}=B_{t} \mathcal{E}_{F}\left(\beta_{v}, \beta_{d}, \beta_{\alpha}\right)
$$

will be correlated as well to the few-nucleon binding energies.

The aim of this work is to study the possible correlation of the nuclear matter binding energy per nucleon with $B_{d}, B_{v}$ and $B_{t}$, in order to improve our understanding of the general and important scaling of observables. Our investigation is based on Eqs. (4) and (5), motivated by our previous discussion that leads to Eq. (11) and in the several works that recognize the role played by the low-energy few-body scales $[8,9,13,12,14,15]$, in defining the observables of few-nucleon systems.

In the present framework, the universal scaling functions connect the proper 


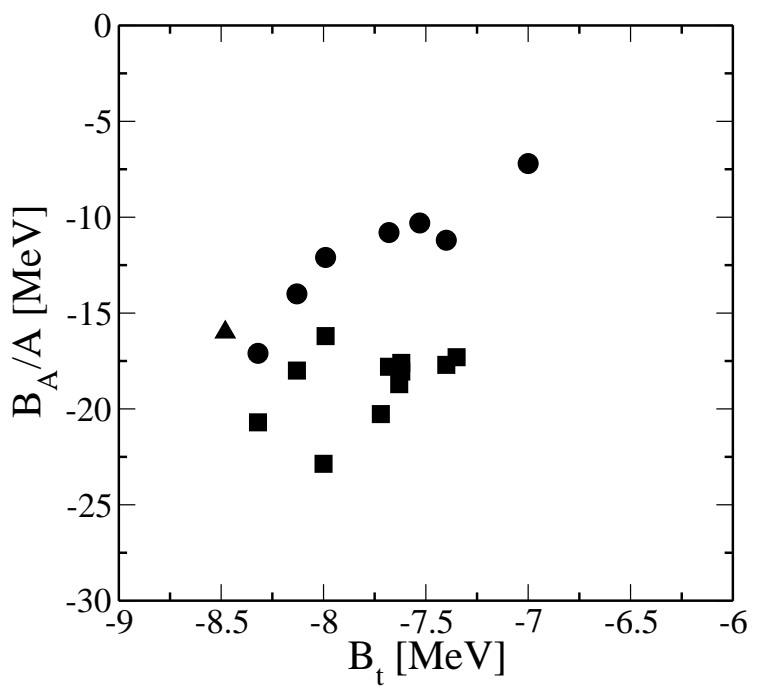

Fig. 2. $B_{A} / A$ as a function of $B_{t}$ extracted from Ref. [25] (solid circles and squares). The squares includes the single particle contribution in the continuum. The full triangle is given by the empirical values.

scales of the few-body system with those of the many-body system, as given by Eqs. (4) and (5). Different potentials, which describe the deuteron and the twonucleon scattering properties give different values of $B_{t}, B_{\alpha} B_{A} / A$ and $E_{F}$. As we have done in deriving Eq. (3) for a class of changes in the short-range part of the nuclear force that keeps the deuteron and low energy scattering properties unchanged, and taking into account that for these variations of the potential the ${ }^{4} \mathrm{He}$ and triton binding energies are strongly correlated as given by the Tjon line, one can rewrite Eqs. (4) and (5) in order to get a one parameter scaling:

$$
\frac{B_{A}}{A}=B_{t} \mathcal{G}\left(B_{t}\right)
$$

for fixed $B_{d}$ and $B_{v}$, where the only true dependence in the class of potential variations is dominated by $B_{t}$. The analogous expression for the Fermi energy is

$$
E_{F}=B_{t} \mathcal{E}_{F}\left(B_{t}\right)
$$

where $E_{F}$ scale with $B_{t}$.

In the perspective of the one parameter functions of Eqs. (66) and (7), it is clear that one could express $E_{t}$ as a function of $E_{F}$ and immediately get

$$
\frac{B_{A}}{A E_{F}}=\mathcal{C}\left(E_{F}\right),
$$




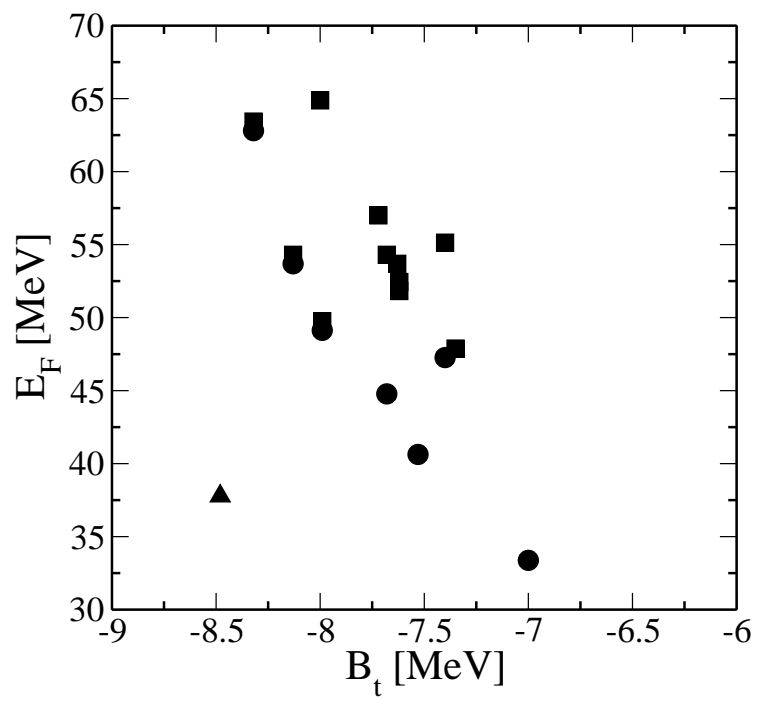

Fig. 3. $E_{F}$ as a function of $B_{t}$ extracted from Ref. 25] (solid circles and squares). The squares includes the single particle contribution in the continuum. The full triangle is given by the empirical values.

the correlation implied by the Coester band.

In order to enlighten our discussion we bring a variety of nuclear matter binding energies $B_{A} / A$ at the corresponding saturation density, represented by the Fermi momenta $k_{F}$, calculated from different two-nucleon potentials. In Fig. 1, we present the well known Coester band in which the results for $B_{A} / A$ and $E_{F}$ are showed. The two distinct bands represent the nuclear matter calculations with and without the single-particle continuum contributions. The empirical values are $B_{A} / A=16 \mathrm{MeV}$ and $E_{F}=37.8 \mathrm{MeV}$ from [27].

The correlation of $B_{A} / A$ with $B_{t}$ expressed by (6) is plotted in Fig. 2, for the same set of results given in Fig. 1, The two-nucleon potentials present different values for the triton binding energy $B_{t}$, while the two-nucleon lowenergy observables are fixed. We observe that the scaling function $E_{t} \mathcal{G}\left(E_{t}\right)$ is quite linear in the interval of about $2 \mathrm{MeV}$ including the triton binding energy. We note that the ratio $B_{A} / A$ depends strongly on $B_{t}$. We understand this fact as a reminiscent manifestation of the three-body scale in the nuclear matter results obtained with only two-body correlations. In Fig. 3, we show the correlation between the Fermi energy and the triton binding energy. In general, we observe that the increase in the three-body scale leads to the increase of the Fermi energy, which is reasonable in view of the scaling function (7). However, we observe that the empirical values disagrees with the general trend of the correlation, a problem that could already be anticipated by looking at the Coester band in Fig. 1,

The inclusion of three-body correlations, which carries the dynamics that stabilizes the Thomas collapse, presumably has a repulsive effect diminishing the 
saturation density and somewhat the nuclear matter binding. Due the shortrange repulsion of the nucleon-nucleon interaction, the nuclear matter tends to saturate at large densities if only two-body correlations are considered and the empirical binding is achieved. The dynamically generated three-body stabilization mechanism carried only through the three-body correlations should appear in the nucleon-nucleon interaction range, however such repulsive contribution is absent if only two-body correlations are considered in the evaluation of nuclear matter properties. Therefore, we suspect that the inclusion of three-body correlations in nuclear matter calculations will bring the correlation curve of the $B_{A} / A$ with $B_{t}$ in Fig. 2 toward the empirical values; and also in Fig. 3, where the saturation densities would be possibly found at lower values for a given triton binding energy.

Recent sophisticated many-body calculations [26], where the triton binding energy and nuclear matter saturation density were adjusted through two threebody parameters make subtle the clear appreciation of the real role of the three-body correlations, once the fit of these realistic forces mixes differences that come from the interaction and from the many-body approximations in nuclear matter calculations. It is beyond our work the discussion of the quite involved many-body approximations needed to perform such calculations. Nevertheless, our conjecture is obviously based on qualitative arguments, which implies that just one three-body scale parameter is relevant for a systematic description of light nuclei and nuclear matter.

The one parameter dependence in Eqs. (6) and (17) suggests to plot the dimensionless quantity $B_{A} /\left(A B_{t}\right)$ as a function of the ratio $E_{F} / B_{t}$, which should look as an almost linear correlation. We display in Fig. 4 the values for $B_{A} /\left(A B_{t}\right)$ versus $E_{F} / B_{t}$. As we could anticipate, the results show a clear linear correlation. We are tempted to say that, if the correlation is extrapolated and assuming that the binding and saturation densities somewhat decreases when three-body correlations are considered, it looks to be possible that the empirical values would be consistent with the correlation band.

In summary, we suggest for the first time a possible scaling of nuclei asymptotic properties, in particular the nuclear binding energies with the triton binding energy, substantiated by recent realistic calculations of light nuclei. This observation generalizes to the many-nucleon context the correlations between observables found in the three and four-nucleon systems. Beyond that, we found that the original correlation between the nuclear matter binding energy per nucleon with the Fermi momentum described by the Coester band can now be seen as robustly represented by the scaling of nuclear matter properties with the triton binding energy. The values of $B_{t}$ carry different aspects of the used two- and three-nucleon potentials. To verify the extension of our conjecture, we propose that one could control the strength of the three-body force without a many-body parameter in order produce different triton binding 


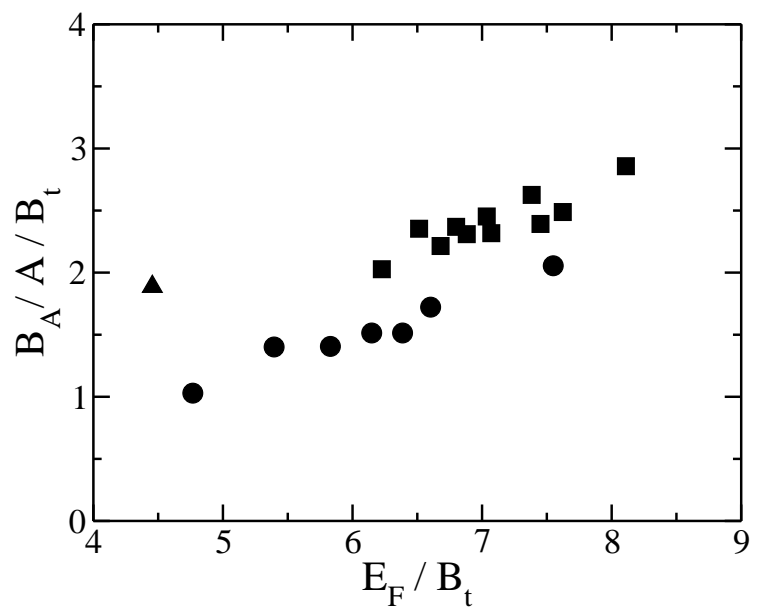

Fig. 4. Infinite nuclear matter binding energy as a function of $E_{F}$, both in units of the triton binding energy. The calculation results are extracted from Ref. 25] (solid circles and squares). The squares includes the single particle contribution in the continuum. The full triangle represents the empirical values.

energies and nuclear matter properties. We emphasizing that the nuclear matter results should be obtained within the same approximations for all models. In this way, we expect that the plotted results in figures 2 and 3 should lie in a very narrow band.

Our discussion may turn in a simple way to systematize results of possible forthcoming realistic calculations for many-nucleon systems. Consistent with our conclusions, it was argued in Ref. [18] that QCD implies that nuclear physics is close to the Thomas-Efimov limit. In this reference, it was conjectured that a small change in the light quark masses away from their physical values could be enough to move the deuteron and the singlet virtual state to zero binding energies. Therefore, nuclear physics could be dominated by longrange universal effective forces making the scalings not only a subtlety but also an evident reality in ab-initio non-relativistic nuclear model calculations.

Acknowledgments. This work was partially supported by Fundação de Amparo à Pesquisa do Estado de São Paulo and Conselho Nacional de Desenvolvimento Científico e Tecnológico. V. S. T. would like to thank FAEPEX/UNICAMP for partial financial support.

\section{References}

[1] W. Glöckle, H. Witala, D. Huber, H. Kamada, J. Golak, Phys. Rep. 274 (1996) 107.

[2] J.A. Tjon, Phys. Lett. B 56 (1975) 217; R.E. Perne and H. Kroeger, Phys. Rev. C 20 (1979) 340; J.A. Tjon, Nucl. Phys. A 353 (1981) 470. 
[3] S.K. Adhikari, A. Delfino, T. Frederico, I.D. Goldman and L. Tomio, Phys. Rev. A 37 (1988) 3666; S.K. Adhikari, A. Delfino, T. Frederico and L. Tomio, Phys. Rev. A 47 (1993) 1093. See also A. Delfino and E.F. Redish in the "Book of Contributions, XIII International Conference on Few-Body Problems in Physics", pg. 304, Edited by I.R. Afnan and R.T. Cahill (1992).

[4] L.H. Thomas Phys. Rev. 47 (1935) 903.

[5] V. Efimov, Phys. Lett. B 33 (1970) 563; Nucl. Phys. A 362 (1981) 45.

[6] J.L. Roberts, N.R. Claussen, S.L. Cornish, and C.E. Wieman, Phys. Rev. Lett. 85 (2000) 728.

[7] D. Blume and C.H. Greene, Phys. Rev. A 66 (2002) 013601; S. Jonsell, H. Heiselberg and C.J. Pethick, Phys. Rev. Lett. 89 (2002) 250401.

[8] S.K. Adhikari, T. Frederico, and I.D. Goldman, Phys. Rev. Lett. 74 (1995) 487; S.K. Adhikari and T. Frederico, Phys. Rev. Lett. 74 (1995) 4572; T. Frederico, A. Delfino, and L. Tomio, Phys. Lett. B 481 (2000) 143.

[9] T. Frederico, L. Tomio, A. Delfino, and A.E.A. Amorim, Phys. Rev. A 60 (1999) R9; A. Delfino, T. Frederico, and L. Tomio, Few-Body Systems 28 (2000) 259; A. Delfino, T. Frederico, and L. Tomio, J. Chem. Phys. 113 (2000) 7874.

[10] A.C. Phillips, Nucl. Phys. A 107 (1968) 109.

[11] T. Frederico, I.D. Goldman, Phys. Rev. C 36 (1987) 1661; T. Frederico, I.D. Goldman, S.K. Adhikari, Phys. Rev. C 37 (1988) 949.

[12] P.F. Bedaque, U. van Kolck, Ann. Rev. Nucl. Part. Sci. 52 (2002) 339.

[13] E. Nielsen, D.V. Fedorov, A.S. Jensen, E. Garrido, Phys. Rep. 347 (2001) 374.

[14] A.S. Jensen, K. Riisager, D.V. Fedorov, E. Garrido, Rev. Mod. Phys. 76 (2004) 215 .

[15] E. Braaten, H.-W. Hammer, cond-mat/0410417.

[16] L. Platter, H.-W. Hammer, U.-G. Meissner, Phys. Rev. A 70 (2004) 052101.

[17] L. Platter, H.-W. Hammer, U.-G. Meissner, Phys. Lett. B 607 (2005) 254.

[18] E. Braaten and H.-W. Hammer, Phys. Rev. Lett. 91 (2003) 102002.

[19] A. Delfino, and T. Frederico, Phys. Rev. C 53 (1996) 62.

[20] S.C. Pieper, Phys. Rev. Lett. 90 (2003) 252501.

[21] S.C. Pieper and R.B. Wiringa, Ann. Rev. Nucl. Part. Sci. 51 (2001) 53; R.B. Wiringa, S.C. Pieper, Phys. Rev. Lett. 89 (2002) 182501; S.C. Pieper, K. Varga, R.B. Wiringa, Phys. Rev. C 66 (2002) 044310.

[22] F. Coester, S. Cohen, B.D. Day, and C.M. Vincent, Phys. Rev. C 1 (1970) 769.

[23] B.D. Day, Rev. Mod. Phys. 50 (1978) 495; B.D. Day, Phys. Rev. Lett. 47 (1981) 226. 
[24] A. Delfino, M. Malheiro, V.S. Timóteo, J.S. Sá Martins, Braz. J. Phys. 35 (2005) 190.

[25] R. Machleidt, Adv. Nucl. Phys. 19 (1989) 189.

[26] A. Akmal, V. R. Pandharipande, Phys. Rev. C 56 (1997) 2261; J. Morales Jr., V. R. Pandharipande, D. G. Ravenhall, Phys. Rev. C 66 (2002) 054308.

[27] R. J.Furnstahl, nucl-th/0504043. 